\title{
Un médecin sur dix cesse son activité auprès des patients
}

\author{
Esther Kraft ${ }^{\mathrm{a}}$, Lisa Loretan ${ }^{\mathrm{b}}$, Nico van der Heiden ${ }^{\mathrm{c}}$ \\ ${ }^{a}$ Cheffe de la division Données, démographie et qualité, $\mathrm{FMH} ;{ }^{b}$ Assistante de projets politique et communication; ${ }^{\mathrm{c}}$ Directeur adjoint / responsable politique \\ et communication de I'ASMAC
}

Combien de médecins abandonnent chaque année leur activité auprès des patients et pourquoi? Une étude représentative mandatée par l'ASMAC et la FMH a examiné ces questions. Il en ressort que $10 \%$ environ de l'ensemble des diplômés annuels en médecine humaine abandonnent leur activité dans le domaine des soins avant l'âge de la retraite. Les motifs les plus souvent invoqués n'ont rien de surprenant: charge de travail, horaires ou mauvaise compatibilité entre vie professionnelle et vie de famille.

\section{Introduction}

La question de savoir combien de médecins choisissent de ne pas exercer d'activité médicale auprès des patients au terme de leur formation a été soulevée à de nombreuses reprises par les médias. Un point fait cependant l'unanimité: il faut autant que possible éviter que les médecins abandonnent la profession, notamment en raison de la pénurie croissante qui s'annonce.

Dans ce débat politique important, les données consolidées concernant le nombre effectif de médecins qui n'exercent plus dans le domaine des soins, les motifs de leur réorientation professionnelle et le sort des personnes ayant quitté la profession font hélas défaut. Soucieuses de disposer de résultats scientifiquement fondés, l'Association suisse des médecins-assistant-e-s et chef-fe-s de clinique (ASMAC) et la Fédération des médecins suisses (FMH) ont mandaté le Büro Vatter et gfs. bern pour mener une enquête et analyser ces questions.

\section{Estimation du nombre de médecins n'exerçant pas}

L'analyse inclut tous les médecins qui ont obtenu leur diplôme de médecin en Suisse entre 1980 et 2009 (N =
22356). L'année 2009 a été choisie de manière à ce que tous les médecins inclus dans l'analyse aient au minimum terminé leur formation postgraduée. Pour estimer la part des médecins qui ont abandonné leur activité médicale auprès des patients avant la retraite, il a fallu procéder en deux étapes: rendre plausible et projeter les chiffres obtenus. Dans un premier temps, la part de l'échantillon de base (les personnes susceptibles d'abandonner leur activité de médecin traitant) a été déterminée en fonction de l'ensemble des personnes interrogées et rendue plausible par une méthode d'analyse multivariée. Cette plausibilisation s'effectue par pondération et «Multilevel Regression and Poststratification (MRP)». Dans un deuxième temps, les recherches ont été complétées par une estimation du "groupe opposé», composé des médecins qui ne présentent pas une probabilité accrue d'abandonner leur activité auprès des patients.

Ces deux étapes ont ensuite permis de modéliser trois scénarios pour la part des médecins n'exerçant plus: D'après l'analyse, la part des médecins qui n'exercent plus est un peu plus élevée chez les femmes que chez les hommes. Suivant le scénario, la part femmes est de 1,2 à 1,6 supérieure à celle des hommes.

Tableau 1: Part des médecins n'exerçant plus auprès des patients, selon le sexe et la région linguistique.

\begin{tabular}{lcccccc}
\hline & Tous & Hommes & Femmes & $\begin{array}{l}\text { Suisse } \\
\text { alémanique }\end{array}$ & $\begin{array}{l}\text { Suisse } \\
\text { romande }\end{array}$ & $\begin{array}{l}\text { Tessin } \\
\text { Scénario bas }\end{array}$ \\
\hline Scénario moyen & $8,4 \%$ & $7,9 \%$ & $9,3 \%$ & $8,8 \%$ & $6,0 \%$ & $7,5 \%$ \\
\hline Scénario haut & $10,7 \%$ & $8,9 \%$ & $12,7 \%$ & $10,3 \%$ & $9,7 \%$ & $9,4 \%$ \\
\hline
\end{tabular}




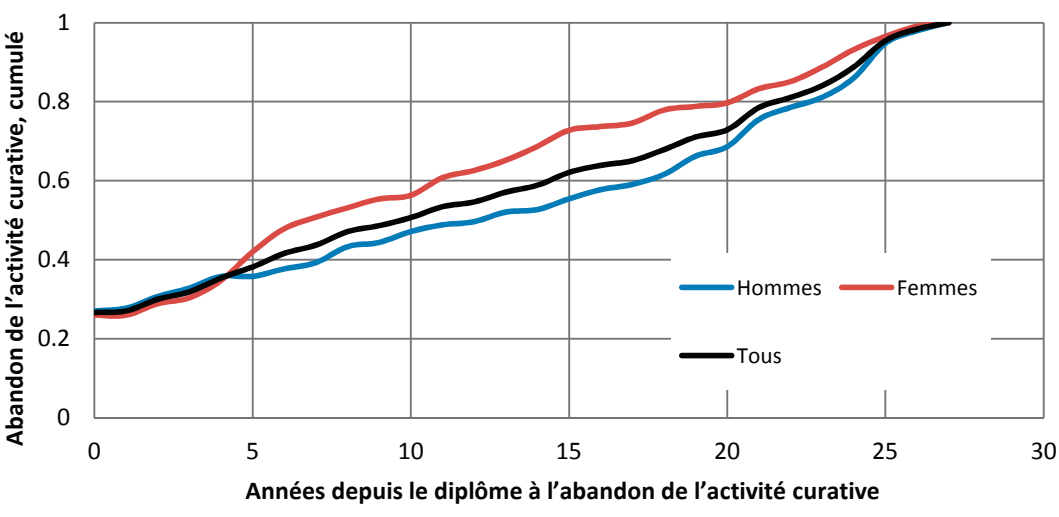

Source: gfs.bern, Laufbahnbefragung von Schweizer ÄrztInnen 2016. N=195: sondés qui ont obtenu leur diplôme de médecin avant 1990 et ont abandonné l'activité curative 27 ans au plus tard après l'obtention du diplôme de médecin. Question 3: «Avez-vous débuté une formation postgraduée au terme de vos études de médecine?» et question 6: «Exercez-vous actuellement une activité auprès des patients (à temps complet ou partiel)?»

Figure 1: Moment de l'abandon de l'activité auprès des patients selon le sexe.

Pour la grande majorité, l'abandon de l'activité auprès du patient est définitif. Selon l'étude, seul un médecin sur dix considère comme plutôt probable ou très probable de reprendre un jour son activité de médecin auprès des patients.

En prenant le scénario moyen, sur les 22536 médecins qui ont obtenu leur diplôme de médecin en Suisse de 1980 à 2009, on compte 2392 personnes qui n'exercent plus dans le domaine des soins. Cela équivaut à environ 80 médecins sur l'ensemble annuel des diplômés en médecine humaine.

\section{Evolution de la carrière médicale}

Sur la base du sondage, nous abordons ci-après plus en détail le moment auquel les médecins ont abandonné leur activité auprès des patients au cours de leur carrière et décrivons par ailleurs comment celle-ci a évolué. Sur l'échantillon $(\mathrm{N}=4023)$, nous avons pu contacter 3333 médecins par courrier postal ou électronique. 1141 interviews ont été réalisées, ce qui correspond à un taux de réponse de $34,2 \%$. L'erreur d'échantillonnage se situe à $\pm 3 \%$.

Pour le groupe des médecins qui ont obtenu leur diplôme entre 1980 et 1989 et qui n'exercent plus dans le domaine des soins, le tableau est le suivant (fig. 1). Si l'on prend les deux sexes ensemble, la courbe présente une augmentation assez linéaire. Cela signifie qu'environ la même part de médecins qui n'exercent plus vient s'ajouter chaque année. Un peu plus d'un quart des médecins quittent la profession avant de commencer leur formation postgraduée pour l'obtention d'un titre de spécialiste. Les courbes sont quasiment identiques pour les femmes et les hommes en début de car- rière. Après environ quatre ans, c'est-à-dire pendant la formation postgraduée, la part des femmes augmente plus vite. Ce n'est qu'environ 25 ans après l'obtention du diplôme de médecin que les deux courbes se rapprochent à nouveau.

Parmi les médecins qui n'exercent pas auprès des patients $(\mathrm{N}=180)$, un quart ont indiqué travailler à l'hôpital ou dans d'autres institutions du secteur de la santé $18 \%$ travaillent dans le domaine scientifique / la recherche, $17 \%$ dans l'enseignement / la formation postgraduée et continue, $17 \%$ dans la promotion de la santé / la prévention, $13 \%$ dans l'administration publique et $13 \%$ dans l'industrie pharmaceutique / la technologie médicale. Les autres médecins sont engagés dans les assurances, les médias, les organisations de médecins, etc.

\section{Quatre types de médecins sans activité auprès des patients}

Si l'on tient compte, d'une part, du moment où les médecins ont abandonné leur activité et, d'autre part, de leur activité actuelle, il en résulte quatre types:

- Type 1: Médecins ayant changé de profession précocement (46\% des médecins n'exerçant plus auprès des patients):

n'ont pas obtenu de titre de spécialiste, leur qualification est utile pour l'activité actuelle.

- Type 2: Médecins ayant quitté la profession précocement (20\% des médecins n'exerçant plus auprès des patients):

n'ont pas obtenu de titre de spécialiste et ne travaillent généralement pas.

- Type 3: Médecins ayant changé de profession tardivement (15\% des médecins n'exerçant plus auprès des patients):

ont obtenu un titre de spécialiste, leur qualification est utile pour l'activité actuelle.

- Type 4: Médecins ayant quitté la profession tardivement (19\% des médecins n'exerçant plus auprès des patients):

ont obtenu un titre de spécialiste et ne travaillent généralement plus.

Un lien statistiquement significatif apparaît clairement: si l'activité médicale auprès des patients est abandonnée avant l'obtention du titre de spécialiste, la qualification médicale obtenue est utile à la majorité de ceux qui exercent ensuite une profession. En revanche, si elle est abandonnée après l'obtention du titre de spécialiste, la qualification médicale obtenue ne présente le plus souvent pas d'avantage direct pour l'activité professionnelle pratiquée. Les hommes réussissent mieux à tirer profit de leur qualification médicale: ils travaillent dans des professions dans lesquelles elle est utile voire 
Charge et horaires de travail

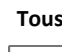

\begin{tabular}{|c|c|c|c|c|}
\hline ous & уре 1 & Туре 2 & Type 3 & Type 4 \\
\hline (34) & 43 & 34 & 37 & 11 \\
\hline 22 & 27 & 32 & 16 & 9 \\
\hline 21 & 27 & (1) & 34 & 5 \\
\hline 16 & 19 & 16 & 13 & 13 \\
\hline 16 & 7 & 23 & 15 & 31 \\
\hline (16) & 22 & 11 & 18 & 4 \\
\hline (15) & 1 & (14) & 4 & 59 \\
\hline 13 & 18 & 14 & (1) & 1 \\
\hline (12) & 12 & 4 & 36 & 2 \\
\hline (12) & (1) & 8 & 26 & 5 \\
\hline (1) & 10 & 20 & 5 & 7 \\
\hline 6 & 8 & 4 & 5 & 6 \\
\hline 6 & 6 & 8 & 5 & 7 \\
\hline 6 & 5 & 3 & 13 & 3 \\
\hline 5 & 8 & 3 & 6 & 1 \\
\hline 5 & 6 & 4 & 3 & 4 \\
\hline 2 & 4 & & 2 & \\
\hline 1 & & 3 & & 4 \\
\hline 6 & 8 & 6 & 3 & 2 \\
\hline
\end{tabular}

Source: gfs.bern, Laufbahnbefragung von Schweizer ÄrztInnen 2016. N = 420: sondés qui ont abandonné l'activité curative avant l'âge de la retraite. Question 21: «Pourquoi n'exercez-vous actuellement pas d'activité curative? Veuillez indiquer les principaux motifs. Veuillez cocher au maximum trois motifs.» Indications en pourcent de la colonne respective (tous, type 1 à type 4).

Figure 2: Motifs de l'abandon de l'activité médicale auprès des patients.

même indispensable, alors que les femmes sont plus nombreuses à ne plus exercer d'activité lucrative.

Parmi tous les médecins ayant abandonné le contact avec les patients, un sur dix environ (9\%) considère comme très probable ou plutôt probable de reprendre un jour son activité auprès des patients. Chez les femmes, $14 \%$ d'entre elles estiment plutôt probable de reprendre une activité médicale contre $4 \%$ chez les hommes.

Les médecins en formation postgraduée, qui exercent donc encore auprès des patients, ont été questionnés sur la probabilité $(\mathrm{N}=204)$ de terminer leur formation postgraduée. $79 \%$ estiment qu'il est probable ou plutôt probable qu'ils obtiennent le titre de spécialiste.

\section{Les motifs de l'abandon de l'activité auprès des patients}

Les médecins qui n'exercent plus expliquent le plus souvent leur abandon par des motifs étroitement liés aux conditions de travail (fig. 2). La charge de travail et les horaires sont de loin cités le plus souvent: environ un tiers des sondés (34\%) considère que ce point compte parmi les trois principaux motifs. La compatibilité de l'activité médicale avec la garde des enfants (22\%) et les contenus du travail (21\%) ont été cités par une personne sur cinq. Le facteur suivant, le niveau d'exigence avec $16 \%$, se réfère au travail à proprement parler. Ce n'est qu'après que viennent s'ajouter des motifs autres tels que la santé, la réorientation ou la retraite (rappelons que l'analyse ne tient compte que des personnes qui ont quitté l'activité curative avant l'âge de 65 ans).

En ce qui concerne la fréquence des différents motifs, le type 1 (médecins ayant changé de profession précocement) ressemble le plus à l'ensemble de toutes les personnes ayant quitté l'activité auprès des patients. Parmi les médecins ayant changé de profession précocement, ce sont même $43 \%$ qui citent la charge de travail et les horaires comme motif important. Le manque de compatibilité de la profession de médecin avec la garde des enfants et les contenus de travail sont également particulièrement souvent cités par $27 \%$ des sondés. Enfin, avec $22 \%$, la réorientation joue aussi un rôle, plus que dans n'importe quel autre type.

Les personnes du type 2 (médecins ayant quitté la profession précocement) citent la charge de travail et les horaires (34\%) presque aussi souvent que le manque de compatibilité avec la garde des enfants (32\%). La santé obtient $23 \%$ et constitue le troisième facteur. Une personne sur cinq de ce groupe cite par ailleurs la carrière de son/sa partenaire comme motif d'abandon.

Pour le type 3 aussi (médecins ayant changé de profession tardivement), plus d'un tiers des sondés (37\%) ont cité la charge de travail et les horaires comme l'un des trois motifs principaux. Pour quasiment le même nombre de sondés (36\%), les perspectives de carrière sont un facteur important; dans aucun autre groupe, ce chiffre est aussi élevé. Avec $34 \%$, les contenus de travail sont également très importants.

Pour le type 4 (médecins ayant quitté la profession tardivement), deux motifs dominent. La majorité (59\%) passe directement à la retraite et près d'un tiers (31\%) invoque la santé.

Chez les femmes, ce sont souvent d'autres motifs qui déterminent l'abandon de l'activité auprès des patients. Elles citent en particulier le manque de compatibilité de la profession de médecin avec la garde des enfants (41\%), alors que seulement $6 \%$ des hommes citent ce motif. Les médecins n'exerçant plus ont également été questionnés sur leur vécu de l'activité médicale. Pour 77\% des sondés $(\mathrm{N}=314)$, la durée du travail était trop longue et pour $68 \%$, les services de piquet trop irréguliers. Les médecins ayant changé de profession précocement (type 1) sont le moins souvent satisfaits de l'activité médicale, les médecins ayant quitté la profession tardivement (type 4) le plus souvent. Un tableau semblable 
Comparaison selon les types et le sexe:

Part des catégories "Compatibilité manifeste» + "Compatibilité plutôt manifeste» Seules les différences de groupes significatives sont présentées.

\begin{tabular}{|c|c|c|c|c|c|c|}
\hline Tous & \multicolumn{1}{c}{ Type 1 } & \multicolumn{1}{c}{ Type 2 } & \multicolumn{1}{c}{ Type 3 } & \multicolumn{1}{c}{ Type 4 } & \multicolumn{1}{c}{ Hommes } & \multicolumn{2}{c|}{ Femmes } \\
\hline 37 & 26 & 30 & 44 & 48 & 43 & 32 \\
\hline 41 & 33 & 40 & 50 & 45 & 48 & 35 \\
\hline 40 & 35 & 40 & 45 & 43 & 45 & 35 \\
\hline 14 & 3 & 8 & 14 & 30 & & \\
\hline
\end{tabular}

Source: gfs.bern, Laufbahnbefragung von Schweizer ÄrztInnen 2016. N=314: sondés qui ont abandonné l'ac tivité curative avant l'âge de la retraite (sans les sondés qui n'ont pas débuté de formation postgraduée). Question 20: «Lorsque vous pensez à votre dernière activité curative: dans quelle mesure votre activité professionnelle était-elle compatible avec vos loisirs, votre couple et votre famille?» Indications en pourcent de la catégorie concernée (tous, type).

Figure 3: Evaluation de la compatibilité.

se présente pour l'évaluation de la compatibilité entre vie professionnelle et vie de famille, cercle d'amis et loisirs (fig. 3). Plus un médecin se décide à abandonner tôt son activité auprès des patients, plus la garde des enfants est un motif prépondérant; un avis que partagent seulement 3\% des médecins ayant changé de profession précocement. Même si aucune différence statistiquement significative ne se dégage entre les sexes dans ce domaine, il semble que ce point motive bien plus souvent les femmes à faire le pas et à abandonner leur activité auprès des patients.

\section{En bref}

L'analyse montre que la part des médecins qui ont obtenu leur diplôme de médecin en Suisse entre 1980 et 2009 et n'exercent plus auprès des patients est estimée entre 8,4 et $12,9 \%$; celle des femmes dépassant légèrement celle des hommes. Pour la grande majorité d'entre eux, l'abandon est définitif - seul un médecin sur dix juge plutôt ou très probable de reprendre son activité médicale auprès des patients. Les hommes réussissent mieux à exploiter leurs qualifications médicales que les femmes lors de leur réorientation.

Les médecins qui arrêtent d'exercer avant l'âge de la retraite se répartissent en trois groupes: un tiers n'a jamais entamé de formation postgraduée, un tiers a abandonné l'activité auprès des patients pendant la formation postgraduée et le dernier tiers après obtention du titre de spécialiste. En moyenne, les médecins qui changent ou quittent la profession précocement, le font entre 30 et 35 ans, ceux qui changent tardivement, le font à un peu plus de 40 ans et ceux qui abandonnent la profession tardivement le font vers 52 ans. Les facteurs suivants sont déterminants pour l'abandon de l'activité auprès des patients:

- Environ un tiers cite comme motif principal la charge de travail et les horaires.

- Le manque de compatibilité de la profession de médecin avec la garde des enfants ou les contenus de travail sont déterminants pour un cinquième des sondés.

- Les médecins qui arrêtent d'exercer ont une vision plus critique de la profession que leurs confrères actifs dans le domaine des soins.

- Les médecins qui n'exercent plus d'activité curative le font en connaissance de cause, c'est-à-dire qu'ils connaissent déjà les défis de la profession au terme de leurs études.

- L'argument d'une situation médicale insatisfaisante n'a pas plus de poids que les autres possibilités professionnelles intéressantes qui se sont proposées.

\section{Conclusion}

Chaque année, sur l'ensemble des diplômés en médecine humaine, la Suisse perd environ 80 médecins qui n'exercent plus auprès des patients. Même si cette part est relativement faible, chaque abandon en est un de trop. Le fait que seulement $9 \%$ des médecins qui ont abandonné la profession puissent s'imaginer reprendre un jour leur activité est particulièrement préoccupant. En d'autres termes, cela signifie que celui qui arrête d'exercer ne reprendra probablement pas. Il est donc d'autant plus urgent de prendre des mesures pour s'assurer que les médecins poursuivent leur activité auprès des patients:

- Les horaires de travail et services de piquet doivent être aménagés de façon plus attractive.

- La compatibilité entre vie professionnelle et vie de famille / garde des enfants doit être mieux garantie et rendue plus visible.

- L'attractivité et les contenus de travail doivent être améliorés. 